\title{
Correction to: Temporal and spatial evolution patterns of drought in China over the past 500 years
}

\author{
Cuixia Wei ${ }^{1} \cdot$ Bing Guo ${ }^{1,2,3,4,5,6,7,8,9} \cdot$ Wenqian Zang $^{7} \cdot$ Fei Yang $^{2} \cdot$ Yuefeng Lu $^{1,2} \cdot$ Dafu Zhang $^{1} \cdot$ Hongwei Wu ${ }^{1}$. \\ Xiaoyan Zhen ${ }^{8} \cdot$ Haorun Xue $^{10}$. Chao Meng ${ }^{5}$. Shuting Chen ${ }^{1} \cdot$ Xiao Yang $^{1} \cdot$ Hailing Zhang $^{1} \cdot$ Xiangzhi Huang $^{7}$. \\ Rui Zhang ${ }^{11}$
}

Published online: 23 November 2021

(c) Saudi Society for Geosciences 2021

Correction to: Arabian Journal of Geosciences (2021) 14: 776

https://doi.org/10.1007/s12517-021-07075-6

In this article the affiliation details for authors were incorrectly given as "Cuixia Wei ${ }^{2}$, Fei Yang ${ }^{2}$, Dafu Zhang", Hongwei $\mathrm{Wu}^{1}$, Shuting Chen ${ }^{1}$, Hailing Zhang" " but should have been "Cuixia Wei ${ }^{1}$, Fei Yang ${ }^{2}$, Dafu Zhang ${ }^{1}$, Hongwei $\mathrm{Wu}^{1}$, Shuting $\mathrm{Chen}^{1}$, Hailing Zhang ${ }^{1}$ ".

The original article can be found online at https://doi.org/10.1007/ s12517-021-07075-6.

Bing Guo

guobingj1@163.com

$\triangle$ Wenqian Zang

zangwq@radi.ac.cn

1 School of Civil Architectural Engineering, Shandong University of Technology, Zibo 255000, Shandong, China

2 State Key Laboratory of Resources and Environmental Information System, Institute of Geographic Sciences and Natural Resources Research of Chinese Academy of Sciences, Beijing 100101, China

3 Key Laboratory of Urban Land Resources Monitoring and Simulation, Ministry of Natural Resources, Shenzhen 518000, China

4 Key Laboratory of National Geographic Census and Monitoring, Ministry of Natural Resources, Wuhan 430072, China
5 Key Laboratory of Land use, MNR, China Land Survey and Planning Institute, Beijing 100035, China

6 Geomatics Technology and Application Key Laboratory of Qinghai Province, Xining 810001, China

7 Aerospace Information Research Institute, Chinese Academy of Sciences, Beijing 100101, China

8 Key Laboratory of Meteorology and Ecological Environment of Hebei Province, Shijiazhuang 050021, Hebei, China

9 Key Laboratory of Digital Earth Science, Institute of Remote Sensing and Digital Earth, Chinese Academy of Sciences, Beijing 100101, China

10 International School, Beijing University of Posts and Telecommunications, Beijing 100876, China

11 Land Satellite Remote Sensing Application Center, Ministry of Natural Resources, Beijing 100048, China 